\title{
Erratum
}

\section{Erratum to: Optical nanocomposites containing low refractive index $\mathrm{MgF}_{2}$ nanoparticles ${ }^{\star}$}

Eur. Phys. J. Appl. Phys. 93, 40403 (2021). https://doi.org/10.1051/epjap/ 2021200298

Leonid M. Goldenberg ${ }^{1,2, *}$, Mathias Köhler ${ }^{2}$, Christian Dreyer ${ }^{1,2}$, Tohralf Krahl ${ }^{3}$, and Erhard Kemnitz ${ }^{4}$

1 Technical University of Applied Sciences Wildau, Hochschulring 1, Wildau D-15745, Germany

${ }^{2}$ Fraunohofer Institute for Schmiedestraße 5, D-15745 Wildau, Germany

3 Nanofluor GmbH, Research \& Development, Rudower Chaussee 29, Berlin D-12489, Germany

${ }^{4}$ Chemistry Department, Humboldt University, Brook-Taylor-Str. 2, Berlin D-12489, Germany

Received: 19 May 2021 / Accepted: 19 May 2021

The address ${ }^{2}$ should be corrected:

Instead of:

${ }^{2}$ Fraunohofer Institute for Schmiedestraße 5, 15745 Wildau, Germany

It should be:

${ }^{2}$ Fraunhofer-Institute for Applied Polymer Research, Research Division Polymeric Materials and Composites PYCO, Schmiedestraße 5, 15745 Wildau, Germany

Cite this article as: Leonid M. Goldenberg, Mathias Köhler, Christian Dreyer, Tohralf Krahl, Erhard Kemnitz, Erratum to: Optical nanocomposites containing low refractive index $\mathrm{MgF}_{2}$ nanoparticles, Eur. Phys. J. Appl. Phys. 94, 30401 (2021)

\footnotetext{
${ }^{\star}$ The online version of the original article can be found at https://doi.org/10.1051/epjap/2021200298

*e-mail: leonid.goldenberg@th-wildau.de
} 